\title{
Entrelacs
}

Cinéma et audiovisuel

Laurent Roth, cinéaste

\section{Squat, désir et Super 8. À propos du Pays fantôme}

\section{Vincent Dieutre}

\section{OpenEdition}

Journals

Édition électronique

URL : https://journals.openedition.org/entrelacs/6158

DOI : 10.4000/entrelacs.6158

ISSN : 2261-5482

\section{Éditeur}

Éditions Téraèdre

\section{Référence électronique}

Vincent Dieutre, «Squat, désir et Super 8. À propos du Pays fantôme », Entrelacs [En ligne], 18| 2021 mis en ligne le 15 novembre 2021, consulté le 14 décembre 2021. URL : http://

journals.openedition.org/entrelacs/6158; DOI : https://doi.org/10.4000/entrelacs.6158

Ce document a été généré automatiquement le 14 décembre 2021.

Tous droits réservés 


\section{Squat, désir et Super 8. À propos du Pays fantôme}

\section{Vincent Dieutre}

D'abord il y a le geste. Le ready-made, l'objet trouvé. Nous sommes en 1914, au bord de la guerre mondiale. Il achète un porte-bouteille au BHV et le promeut œuvre d'art. Non. C'est sur eBay juste avant les années Covid, et c'est une bobine Super 8 qu'il achète. Pas cher. Laurent ne sait pas trop ce qu'il y a dessus : une caserne ou quelque chose comme ça. Ce pourrait être un film des années 1960, 1970. Ça n'a pas grande importance. Il nous montre la bobine une fois, sans son, sans rien. Elle l'obsède. Les casernes l'obsèdent. Obsèdent ses films. Comment squatter celle-ci, l'habiter en cinéma. La Bobine pose une question, appelle une, deux, trois, quatre ou cinq réponses ou questions en retour.

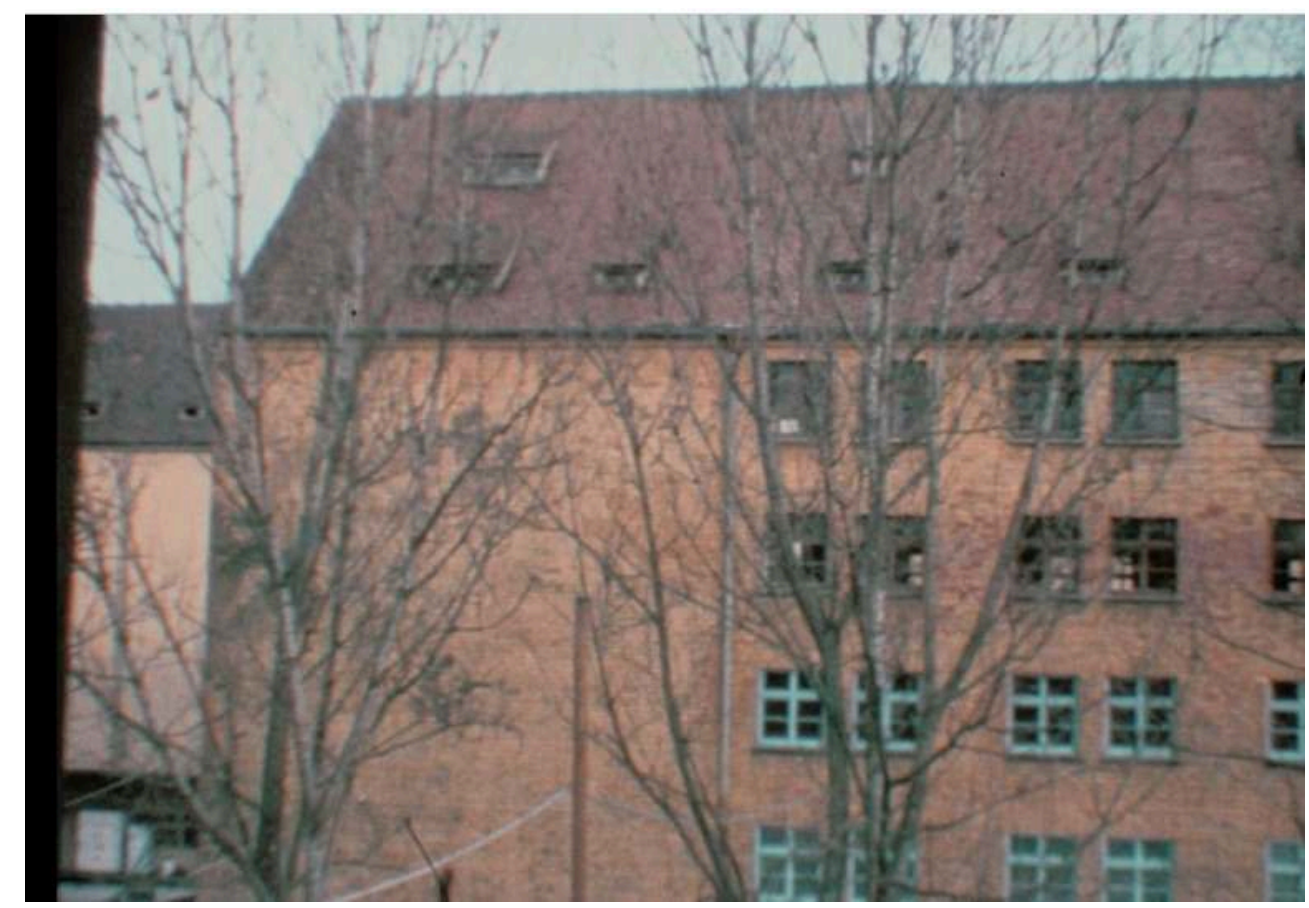


2 Laurent au sommet de son art. Archiviste des imaginaires qu'il prête aux photogrammes, aux images blanches même. Pleurons Christian Boltanski et les rayures et les poussières et le grain du Super 8 perdu pour toujours comme Pondichéry. Il aime revenir là-dessus Laurent, la perte, l'indicible compassion qu'on peut ressentir parfois pour les héros dérisoires. C'était un faible pour les consulats perdus du Gange chez Marguerite, c'en sera un pour les bataillons d'Afrique égarés dans le désert (option tartares), en transit dans des casernements de Province, puis sur eBay d'Halong chez Laurent Roth. Alors la bobine, il lui donne corps, il lui prête voix.

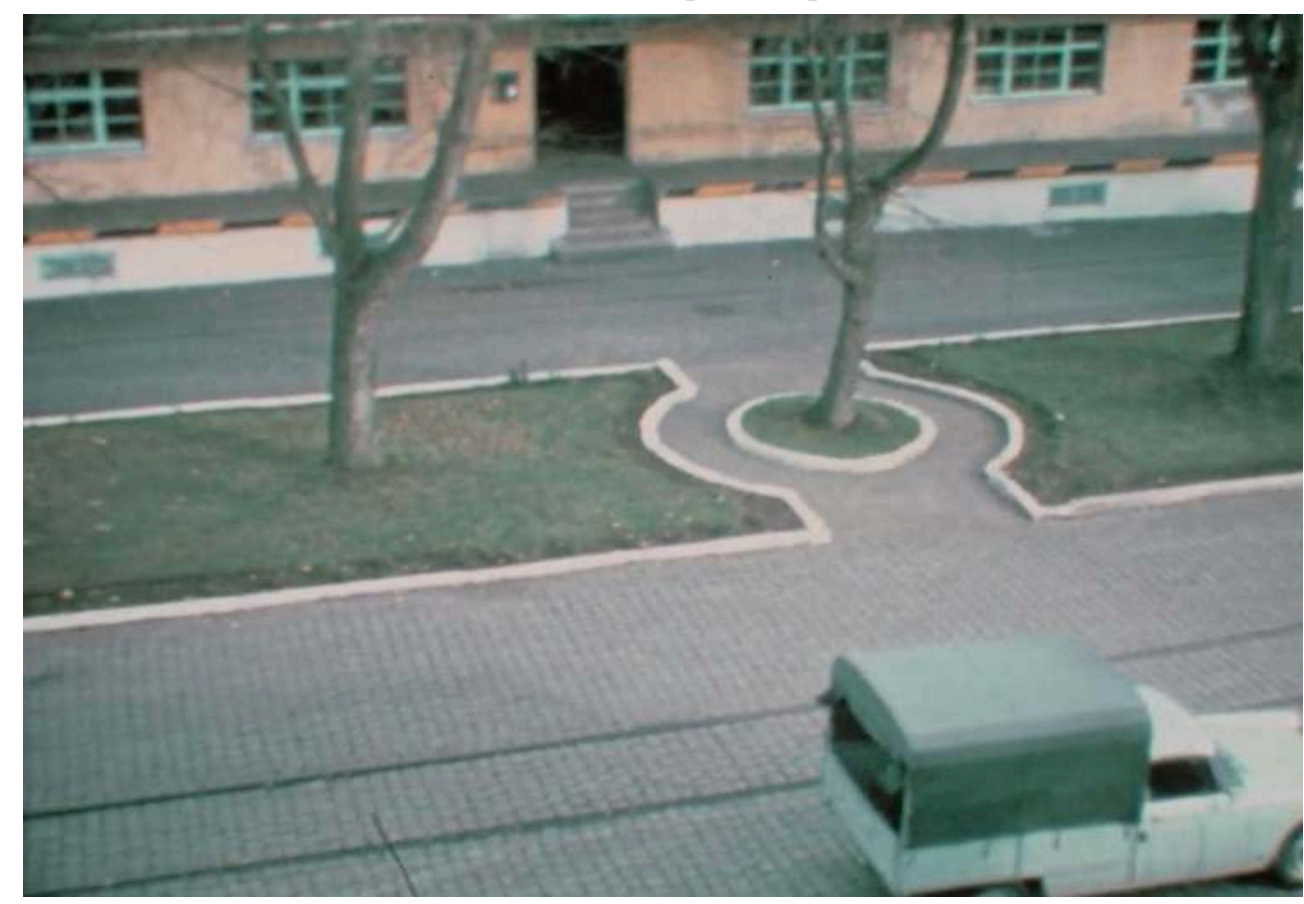

Le ready-made se corrige, se socle de littérature, se scénarise. Piste 1:1964, on rentre d'Algérie, ou du Djebel. Chacun tente d'oublier, de faire comme si rien n'avait été, mais une mémoire se rebiffe, les corvées de bois ne passent pas ; Laurent était là qui a tout noté dans son journal, les noms propres, la bagarre qui s'amorce, l'oubli qui ne viendra jamais. Image blanche. La caserne de province, ou même d'une Algérie du Nord improbable. La gouttière de traviole (une chute ?) clôt la piste. La sale guerre, la torture, qui refuse le chutier et qui erre sur eBay en quête d'un geste d'artiste, d'un film d'histoires de Laurent Roth. 


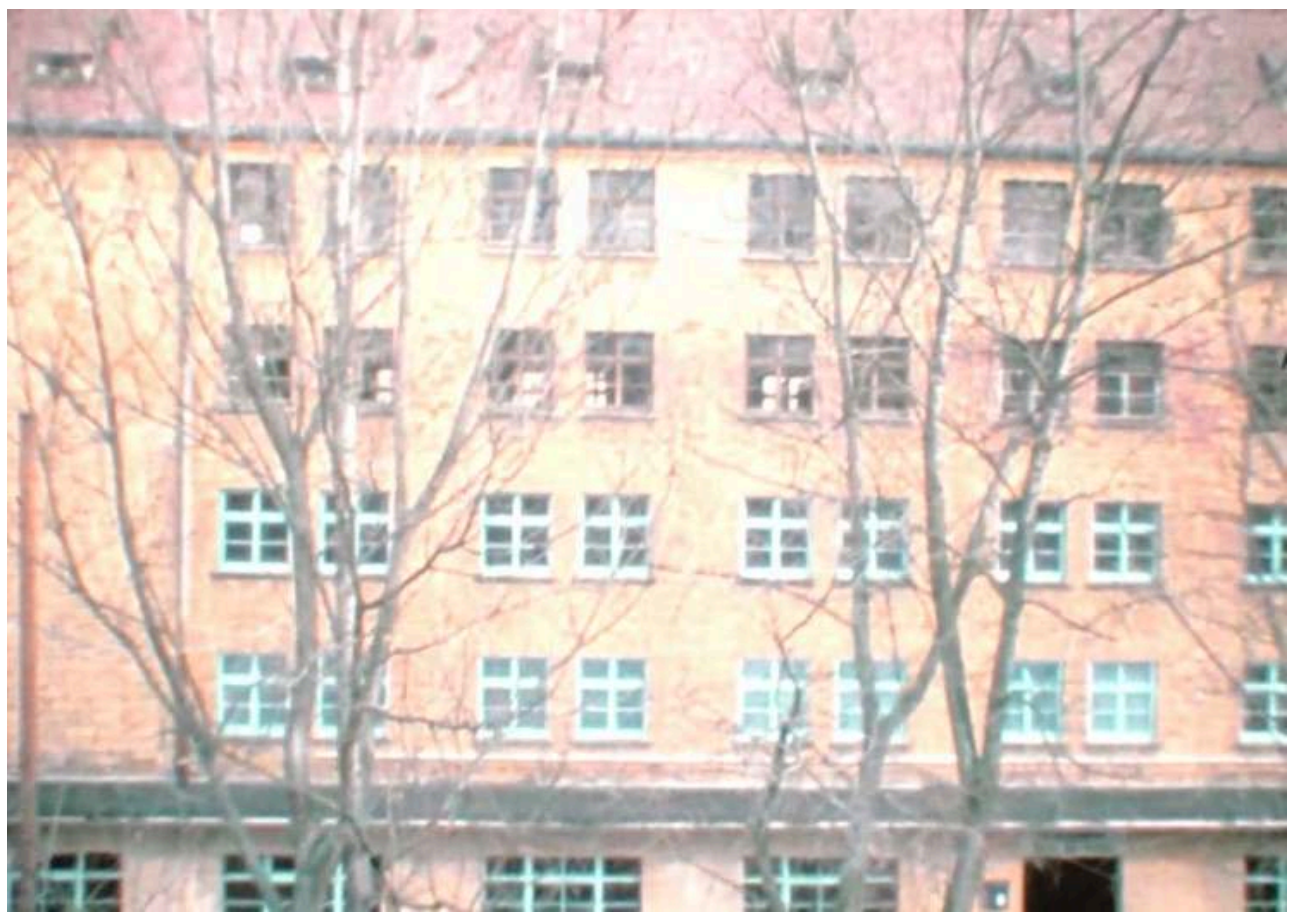

4 Comme moi, Laurent est né pendant « les événements ». Comme moi, son enfance s'est lézardée de ces Vietnams aphones et lointains, dont les seuls signes chauds étaient les lettres OAS qui affleuraient sur les murs gris du gaullisme. Rien de nostalgique dans le cinéma de Laurent Roth, mais le désir fou de rétablir la complexité d'un climat comme seul peut le faire le cinéma, du moins le cinéma qu'il défend. On pense au Combat dans l'île (1962) d'Alain Cavalier, ou au Petit Soldat de Godard (1963) tournés dans ce climat-là, qu'il ne s'agit pas de célébrer ou de juger, mais d'admettre. Quitte à envoyer un ange (Mireille Perrier) questionner ceux qui s'y sont brûlé les yeux (Pierre Schoendoerffer), il y aura film (Les Yeux brûlés, 1986) et ressassement-étirement-ralentissement du même film indéfiniment. Mais c'est dans Le Pays fantôme (2019), que cette quête un peu folle s'installe au mieux, je crois. Comme chez des Pallières ou chez les Gianikian, l'urgent est de déjouer les pièges de la synchronie, de la scénarisation, pour renouveler le contrat de croyance avec le spectateur. Régler son compte à la vraisemblance pour faire scintiller une vérité, insaisissable, hybride, foireuse, mais incroyablement efficace. Et le film, Le Pays fantôme que cartographie Laurent, se fait variation sur un thème postcolonial, installation (plus Porte Dorée que Palais de Tokyo), chaque piste ne menant nulle part qu'au noir qui la sépare de la suivante (après la gouttière de traviole, le plan en trop). 


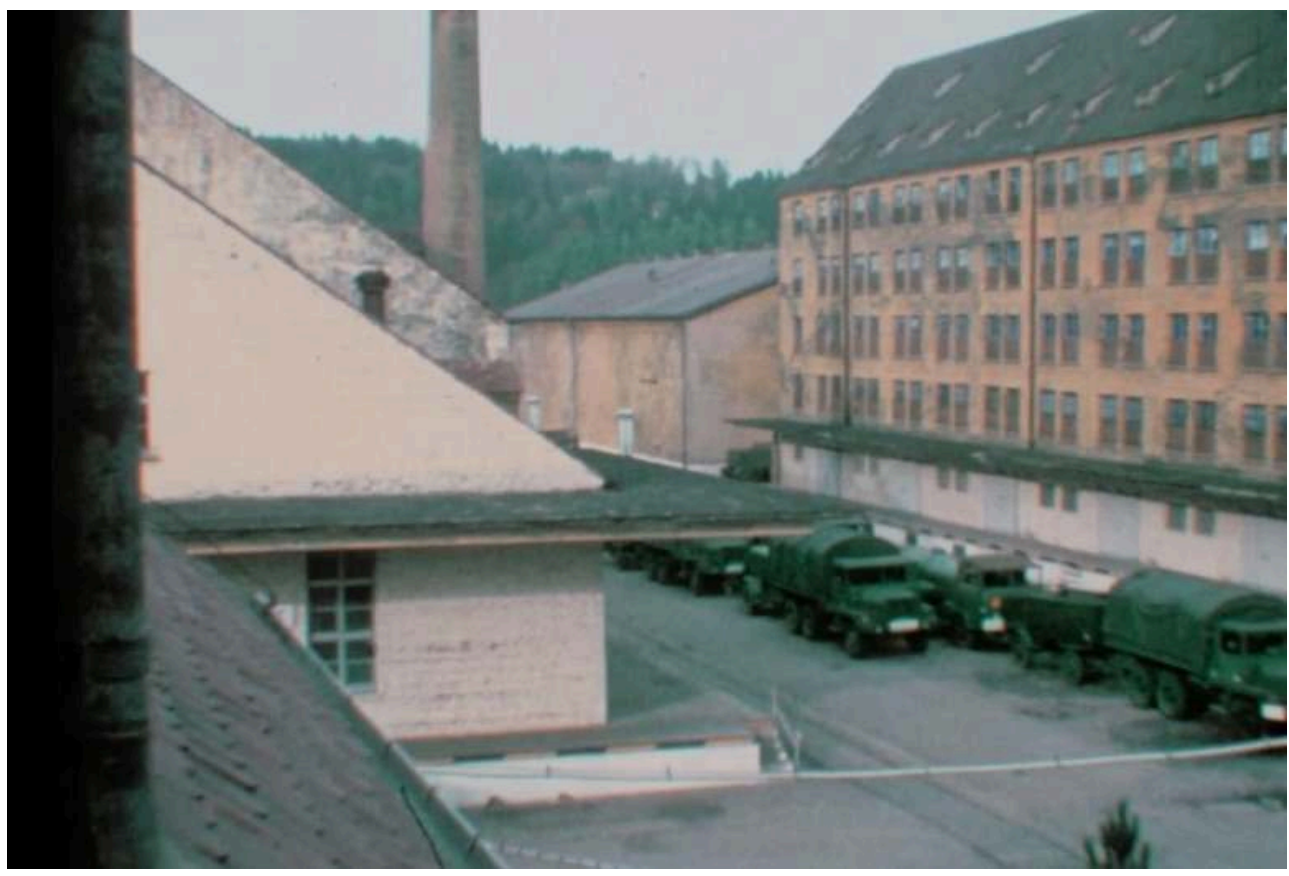

5 La piste 2, c'est le désir. Le troufion a sa mousmé et s'enferme avec elle les jours de désoeuvre. La caserne est là, toujours nulle part, toujours déserte. La fille vient de partir et le gars n'en peut mais. Il sait que ça ne va pas durer. Alors il déballe sa quincaillerie Pépé-le-Moko, son jeu de cartes postales orientalistes et coquines, tout de soumission et d'odeurs exotiques. C'est lui qui est piégé dans ce temps hors l'histoire qui n'est doux que parce qu'il est furtif, illicite, délicieux. Double vénéneux de la gégène, ce temps hantera la mémoire et le désir, comme les bobines Super 8 hantent les «Bons Coins » du réseau. Est-ce le même appelé que celui de la piste 1 ? Peu importe, la voix est la même de pistes en variations, la voix de l'archiviste-cinéaste Laurent Roth qui épouse goulûment les méandres lascifs de son récit, les colères des uns, l'introspection des autres, le silence même.

6 Car la brièveté du geste, la liberté du ready-made, la rigueur du dispositif, permettent aussi à Laurent, le temps d'une bobine, de quitter la légèreté polie et délicate de ses autofictions. Le sentiment tragique de la vie qui sous-tend tout son travail s'incarne plus frontalement au Pays fantôme. Bien sûr J'ai quitté l'Aquitaine ou même la récente Nymphe Scylla nous content bel et bien l'arrachement à l'enfance comme catastrophe centrale, mais se protègent aussi d'un élégant voile rohmerien, tissé d'ironie douce et de références amusées, à la manière distante d'un Emmanuel Carrère ou du premier Moretti. Ici, au Pays fantôme, l'histoire est à vif, et Laurent invite et invente d'autres vies que la sienne pour squatter en «clandestin » la caserne Super 8 sans histoire, tandis que ce tragique par effraction surgit entre l'image et le son, au rythme implacable des variations. 


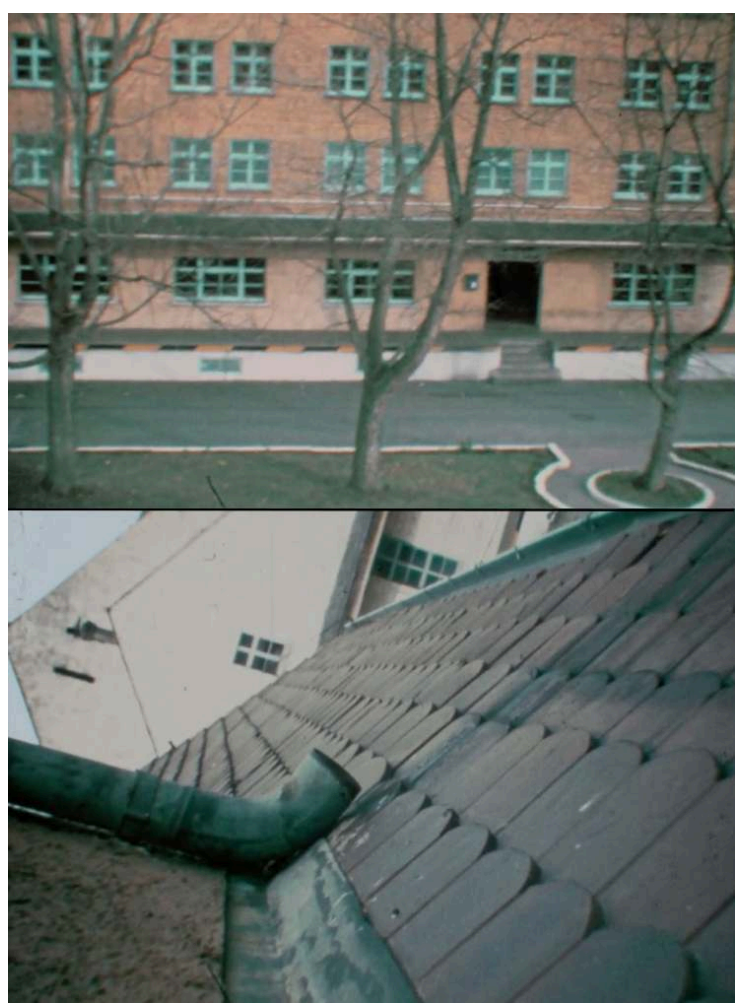

7 La piste 3 est un genre de climax rothien. On entend un muezzin au loin. Autre souvenir d'un autre troufion? d'une autre caserne (plus blanche, plus au sud) ? Peut-être. On s'en balance. Souvenir d'une rencontre spirituelle au sommet, petite pause recueillie au beau milieu du désastre. On avait aménagé une petite mosquée dans un coin de la caserne, et l'appelé, tout catholique qu'il est, est venu prier là. Un autre arrive, musulman lui, qui va joindre sa prière à la sienne, partager son silence et son petit carré de foi pure dans la caserne en sursis. Le temps de la violence, comme celui du sexe, se suspendent un instant dans le trou d'air d'une prière commune, d'une rédemption possible. Là encore, Laurent aborde plus directement cette foi qui l'anime depuis toujours, solide et hospitalière. Pas la piété des monts de piété, la foi de Laurent Roth est de celle qui rend l'art indispensable et qui le fait savoir.

8 La 4 est une vraie-fausse piste. Mise en abîme à la Godard, comme pour éprouver la règle du jeu. Laurent ne protège plus ses sources. Il visionne la bobine. Il la commente en direct. Il fera avec. Quelles infos nous livre-t-elle. Un lieu ? N'importe où aux abords d'une grande ville française, au nord du nord d'Alger la Blanche en tout cas, à moins que ce ne soit dans l'Allemagne occupée. Il a montré le film à d'autres, à l'Autre, pour voir, pour renseigner l'énigme. Mais rien, on le trouve banal, un peu chiant même. On y accroche difficilement une Algérie quelconque, mais lui, le clandestin, le voleur, ça lui va : un non-lieu ingrat qui rappelle le temps du service militaire obligatoire et qu'on n'aurait pas encore transformé en centre d'art contemporain. Pour finir, Laurent a reconnu un modèle de voiture et peut dater le film. 1978. Derniers feux du Super 8 et des casernes comme lieu de mixité sociale. Au cas où nous aurions cru à un exercice oulipien d'épuisement de la fiction, Laurent casse son jouet et nous renvoie au vide béant des images, à la gouttière de traviole en postlude exténué, nous rappelant à la réalité du dispositif cinéma, comme on exfiltre des touristes du Pays fantôme. 


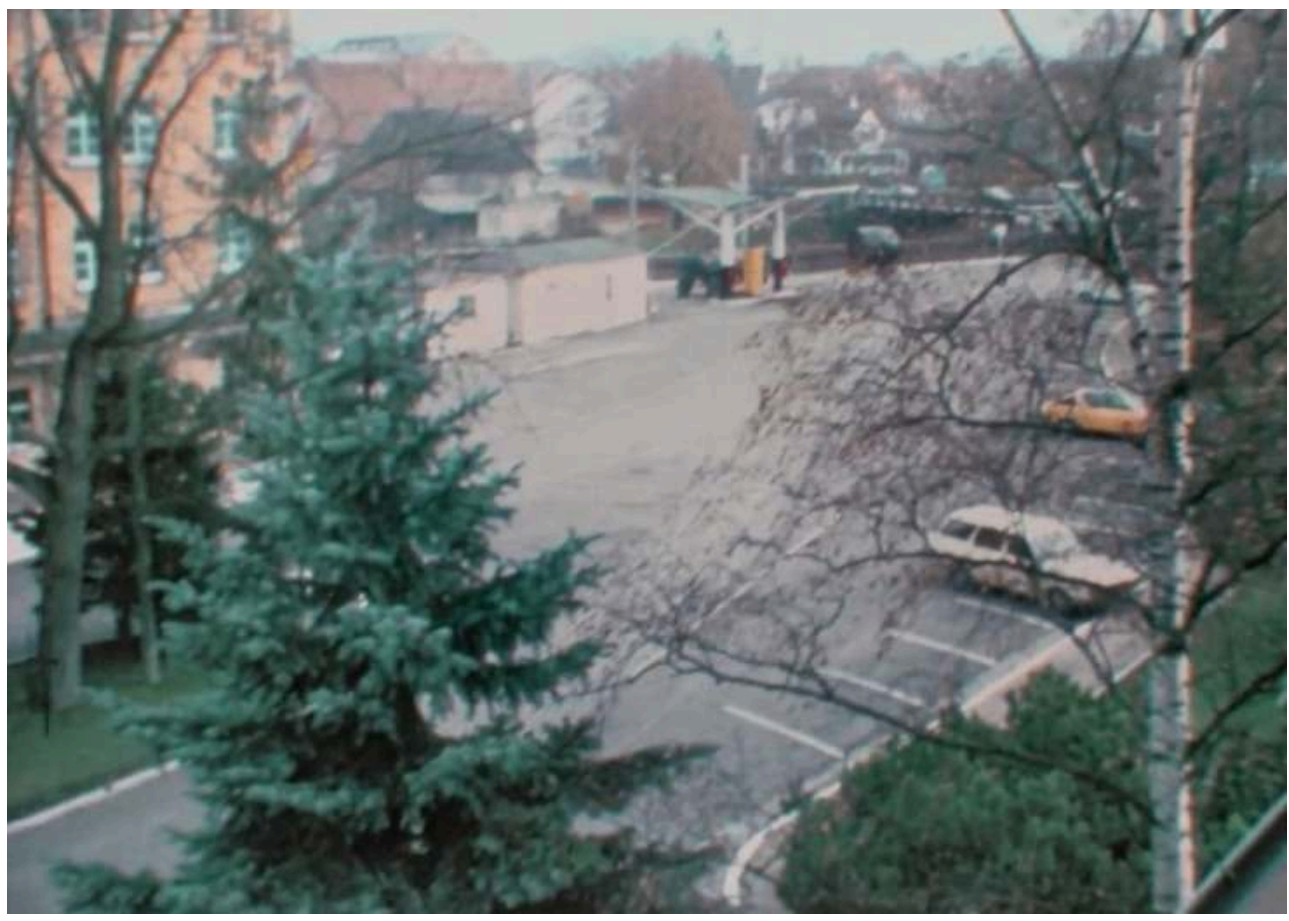

9 Le dernier possible a tout d'une apothéose. C'est la mort de l'auteur et Laurent n'y est plus pour quoi que ce soit. Nous sommes en 1982 et Jeff Koons va acheter des aspirateurs à Canal Street. Non! c'est ici et maintenant et Laurent Roth achète sur eBay une bobine Super 8 dont il ne sait pas grand-chose; une caserne française, en Allemagne, 4 euros. Il aurait pu se la jouer minimal chic et clore comme il avait commencé par la bobine à nu, sans fiction corvée de bois, sans plus d'amante de la Kabylie du sud, ni de mosquées œcuméniques, sans boniments ni pirouette brechtienne, sans rien que l'âpre beauté du geste duchampien en phase terminale. Oui mais... Non. Et c'est là que le cinéma de Laurent continue de surprendre: nous reverrons tout encore une fois, de la caserne improbable à la gouttière de traviole, des camions aux jardins mités en passant par le modèle 78 de la voiture jaune, mais cette fois, l'arrogance narrative se retirera, le silence frémissant du projecteur se taira pour laisser place à l'assomption foudroyante de la musique. Alors, la beauté violemment mélancolique des accords de Schumann mangera tout, du trouble de l'archive au questionnement de l'Histoire. Le film se love dans son devenir-musique, loin de toute musique de film. Et Laurent Roth célèbre en toute modestie, religieusement, une bonne fois pour toute, la puissance encore vivante du cinéma. 


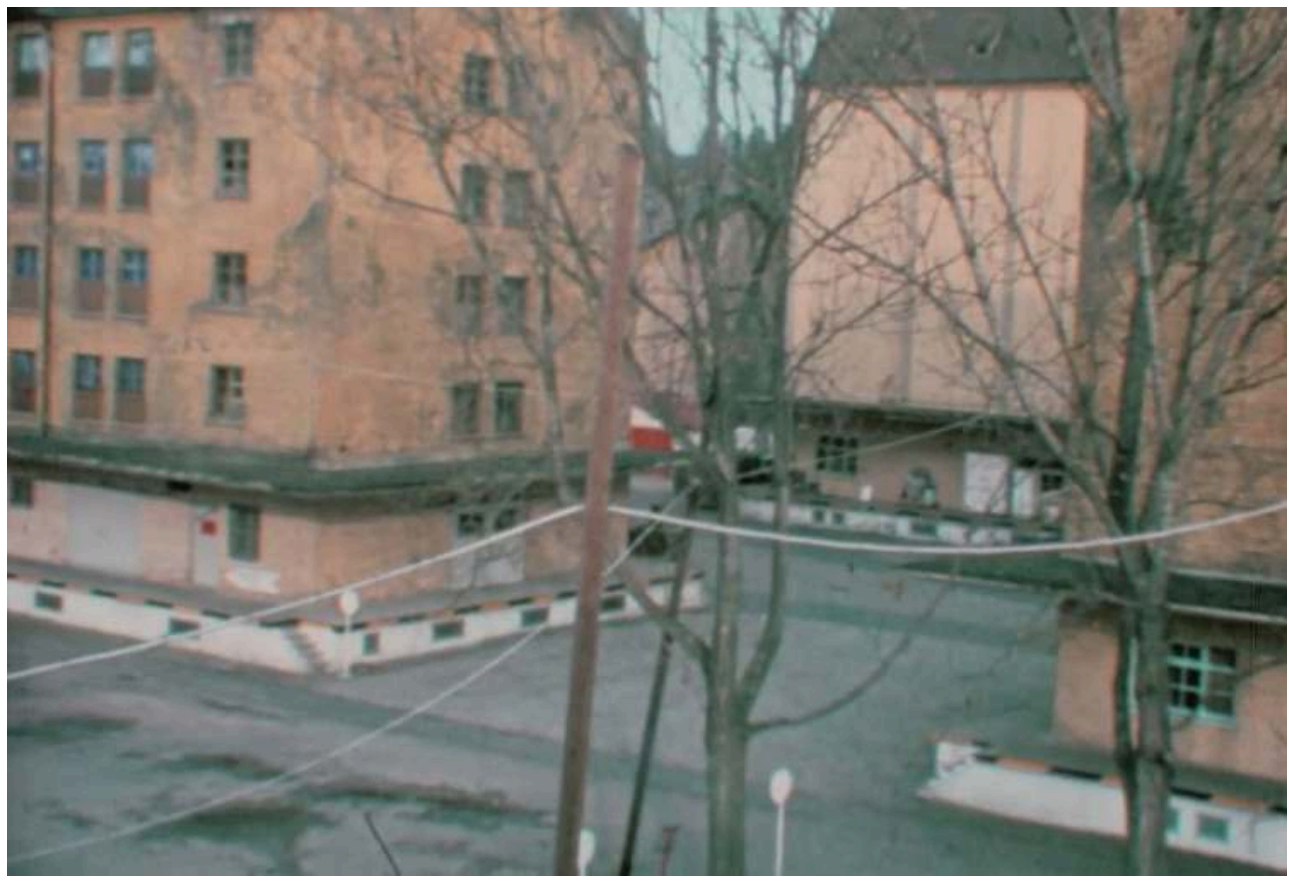

Crédit images : Laurent Roth

\section{RÉSUMÉS}

Le Pays fantôme, c'est une bobine Super 8 que Laurent achète pas cher, sur eBay. Il ne sait pas trop ce qu'il y a dessus : une caserne ou quelque chose comme ça. Ce pourrait être un film des années 1960, 1970. Ça n'a pas grande importance. Il nous montre la bobine une fois, sans son, sans rien. Elle l'obsède. Les casernes l'obsèdent. Obsèdent ses films. Comment squatter celle-ci, l'habiter en cinéma...?

Le Pays fantôme is a Super 8 reel that Laurent buys cheaply on eBay. He doesn't really know what's on it: a barracks or something like that. It could be a film from the 1960s or 1970s. It doesn't really matter. He shows us the reel once, without sound, without anything. He is obsessed with it. The barracks obsess him. Obsesses his films. How to squat this one, to inhabit it as a cinema...?

\section{AUTEUR}

\section{VINCENT DIEUTRE}

Ancien élève de l'IDHEC et lauréat de la bourse « Villa Médicis Hors les Murs », Vincent Dieutre a résidé à New York et à Rome avant de se consacrer à la réalisation. Il explore les limites du documentaire et de l'autofiction, alliant l'intime au collectif dès Lettres de Berlin (1988) puis dans ses longs métrages, notamment Rome désolée (1995), Fragments sur la Grâce (2006) et son cycle de films d'Europe (Leçons de Ténèbres, 2000 ; Mon Voyage d'Hiver, 2003) ou encore, plus récemment Jaurès (2012) et Berlin Based (2019). Passionné par les rapports entre cinéma, littérature et art 
contemporain, Vincent Dieutre a cherché à les redéfinir dans ses écrits critiques (La Lettre du Cinéma, Politis, Mixt(e)) à travers l'expression « tiers-cinéma ». 